\title{
Effective Parameters on Hardness Behavior in Nanoindentation of Nylon66/Clay Nanocomposite Using Representative Volume Element
}

\author{
A. Soleimani and M. Saadatfar
}

\begin{abstract}
Finite element method (FEM) has been widely used for numerical simulation of hardness tests on bulk material in order to analyze its deformation response and investigate the influence of indenter geometry, friction and material elastic and plastic properties. The present work is to develop representative volume element method for modelling of nanoindentation behavior of exfoliated Nylon66 /clay nanocomposite that has nonlinear elastic behavior using numerical simulation. A two dimensional simulation is done and the effect of indenter and specimen geometry on the response of hardness test is investigated.
\end{abstract}

Index Terms-Finite element, polymer/clay, nanocomposite, representative volume element method, spherical indenter.

\section{INTRODUCTION}

Since many technologies have moved to ever smaller scale, characterization of the intrinsic mechanical properties of materials has become more difficult and complicated. Polymer/clay nanocomposites are polymeric materials that are reinforced by nanoclay particles whose dimensions are in the sub-micron scale and the particles are composed of stacks of $1 \mathrm{~nm}$ thick mono-layers whose in-plane dimensions range from $100 \mathrm{~nm}$ to $1000 \mathrm{~nm}$. To find applications for this new class of materials, their mechanical properties have to be sufficient to ensure mechanical reliability.

All of the analytical models such as Mori-Tanaka or Halpin-Tsai are used for predicting the elastic modulus and some other parameters of nanocomposites. As we know these models have some limitation in predicting the nanocomposite properties and using them for predicting the nonlinear mechanical behaviour nanocomposite is questionable.

Nanoindentation testing at very low load is a successful technique for studying the mechanical properties of these materials.

Finite element method (FEM) has been widely used for numerical simulation of hardness tests on bulk material in order to analyze its deformation response and investigate the influence of indenter geometry, friction and material elastic and plastic properties.

In recent years, the study of polymer/clay nanocomposites has attracted major research and commercial interests. The Toyota group [1]-[3] was the first to achieve successful exfoliation of clay in Nylon 6 through in situ polymerization. They have shown that inserting as little as $4.7 \mathrm{wt} \%$ clay into Nylon 6 doubles both elastic modulus and strength. However,

Manuscript received November 23, 2011; revised December 30, 2011.

A. Soleimani and M. Saadatfar are with the Department of Mechanical Engineering, Najafabad Branch, Islamic Azad University, Isfahan, Iran (e-mail:soleimani@pmc.iaun.ac.ir; msaadatfar@ut.ac.ir). it is the functional properties of nanocomposites that are the main driving force in nanocomposite development. The nanoindentation test is one of the important tests for understanding the behavior of materials and many researchers tried to simulate it for bulk material and thin films. The indentation of a flat surface with a rigid axisymmetric body is known to be one of the most complicated mechanical problems, with no analytical solution. FEM is then helpful to understand the mechanical behavior of the surface during loading and unloading phases. Some researchers tried to simulate nanoindentation using FEM [5], Also the effective parameters (such as friction in contact surface, Influence of indenter tip) in nanoindentation process and simulation of it, is investigated [6]. In another work, an algorithm for achieving the stress-strain curve from simulation of nanoindentation is improved [7]. In literature there are some experimental works on nanoindentation of Polymer/clay nanocomposites with Nylon 66, Nylon 6 and Nylon 11 matrix to understanding the properties of them [8]-[10]. As it can be seen many researchers tried to simulate indentation and nanoindentation test on composites and bulk materials but any simulation of nanoindentation process on a nonlinear Polymer/Clay nanocomposites has not be seen.

The purpose of the present work is to modeling the spherical nanoindentation process of exfoliated nanocomposite by numerical simulation. First the tensile test on a three dimensional representative volume element (RVE) is simulated and stress-strain curve is obtained. Then by using this stress-strain curve, the nanoindentation process of nanocomposite is simulated as an isotropic material. Finally, the effect of indenter radius and friction coefficient is investigated. Furthermore, it is observed that the simulation results of nanoindentation depend greatly on the mesh size but very little on the friction coefficient of indenter and specimen.

\section{Finite ELEMENT Modeling}

A 3D finite element model of a single group of aligned clay flakes embedded in a polymer matrix was developed. The model assumed three linear elastic isotropic material phases: a polymer matrix phase, a clay flake inclusion, and an interphase connecting the matrix and inclusion. The microstructure for the finite element micromechanics model was simplified by assuming the clay flakes in a single group were parallel to each other. The model assumed the clay flakes were square and perfectly bonded to an interphase, with both the clay and interphase suspended in a polymer matrix.

The data used in this study for Nylon66 are from literature 
[5], [6]. The stress strain curve used for Nylon66 is shown in Fig. 1 also clay is assumed linearly elastic [4].

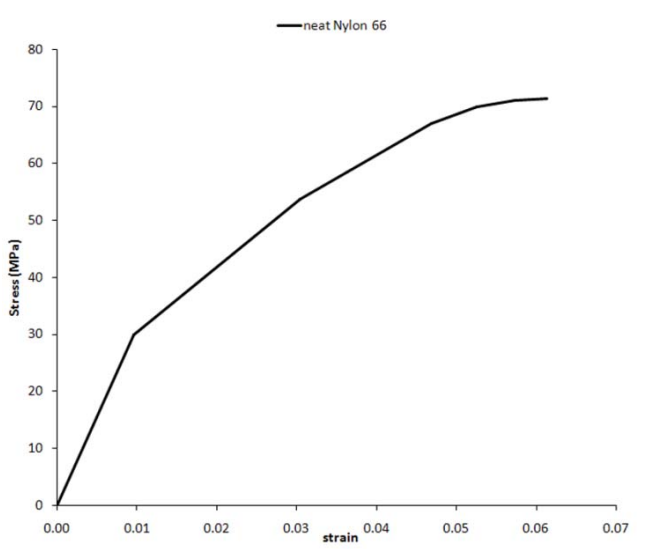

Fig. 1. Stress-strain curve of neat Nylon66.

The clay's elastic modulus is $50 \mathrm{GPa}$, and also the matrix and clay Poisson's ratios are 0.35, 0.2 respectively [4], [6]. The aspect ratio of the particles is assumed to be 100. In model it is assumed that both the matrix and the clay particles are isotropic and the particles are perfectly bonded to the matrix. The model is subjected to plane stress. A uniform strain is applied to one edge of the model by applying a point force on a rigid reference node that is kinematically coupled with the loaded edge in the axial direction so that nodes along the loaded edge have to exactly follow the displacement incurred by the reference node. The stress is calculated by dividing the reaction force applied to the reference node by the area of the loaded edge. This procedure is better than applying a uniform displacement directly to the edge of the model, as the calculation of the average stress would be more cumbersome.

Individual particles are merged with the matrix to make up the composite but yet retaining the geometrical boundaries with the matrix. The model after merging is a single entity but the elements forming the particles have different properties to those of the matrix. The meshes are shown in Fig. 1. The model was meshed using 8-node linear brick elements with reduced integration and hourglass control. The model meshing was organized to maintain 8-node rectangular, or near rectangular, versions of this element throughout the volume.

The stress strain curve is obtained point by point in the elastic region and is assumed to be linear after yield stress. In this stage, the stress-strain curve obtained from previous simulation is used for nanocomposite.

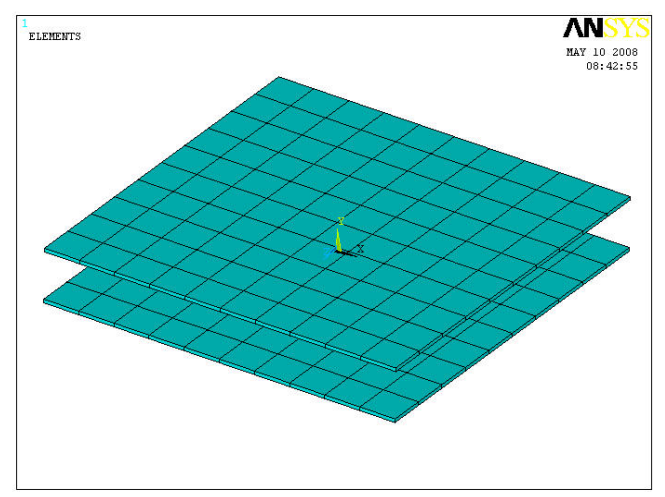

(a)

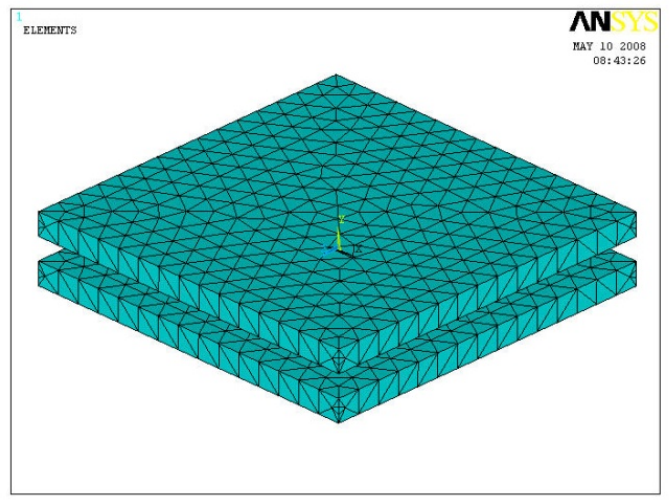

(b)

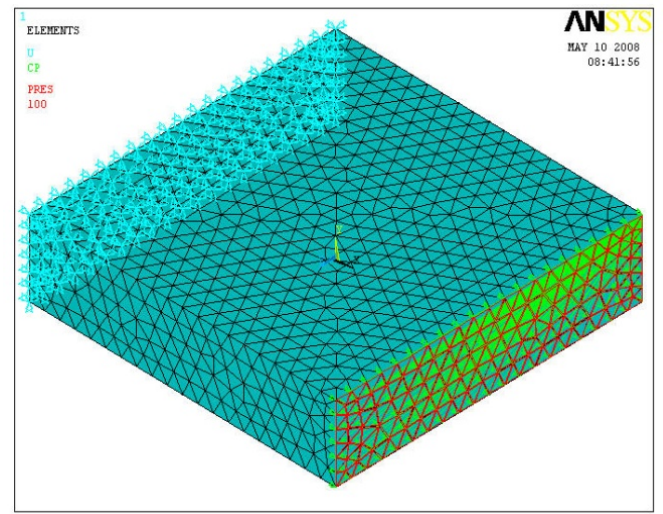

(c)

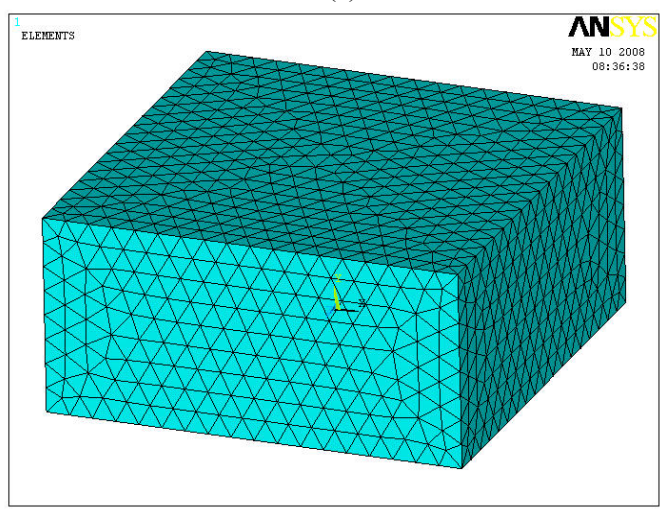

(d)

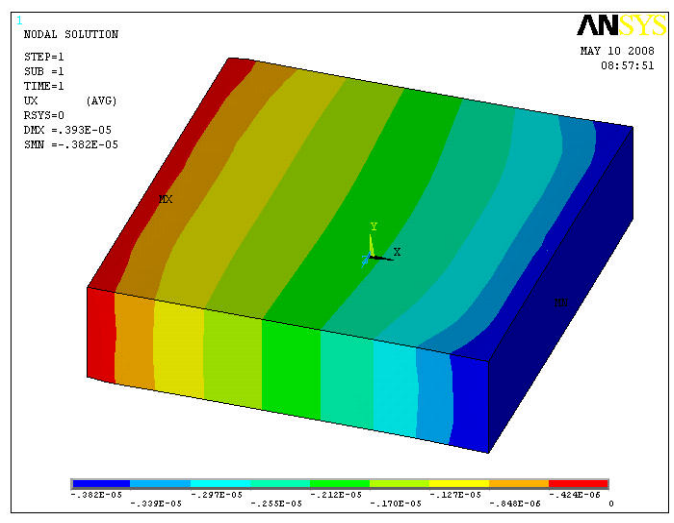

(e)

Fig. 2. Finite element model and meshes.

For simplicity in modeling the nanoindentation process, an 2D model is presented. An ideally spherical indenter is used. The friction coefficient between the tip and the specimen surface is assumed to be zero. To model the specimen, a high order two-dimensional element is used having two degrees of freedom at each node with compatible contact surface elements. The imposed geometrical boundary conditions are: 
the nodes on the revolution Y-axis can move only along this axis, although all the nodes on the bottom of the mesh are fixed in Y-direction and can move in the radial direction. The boundary condition is shown in Fig 3. It is known that the region of interest, which is in the vicinity of the indenter tip, is very small compared with the overall specimen size. Accurate FE simulations require fine mesh in the region of interest. However, it is unrealistic to use this fine mesh for the entire specimen. Therefore, the mesh is continuously coarser further away from the indentation region.

Two subsequent steps, loading and unloading, were used to simulate the whole nanoindentation procedure. During loading, the rigid cone indenter moves along the y-axis and penetrates the specimen to a preset maximum depth; during unloading, the indenter returns to its initial position. For a given indenter displacement, the corresponding load determination was achieved by summing the reaction forces on the bottom nodes. In order to obtain stable and convergent results at each depth increment, nonlinear iterations according to specified convergence criteria is performed.

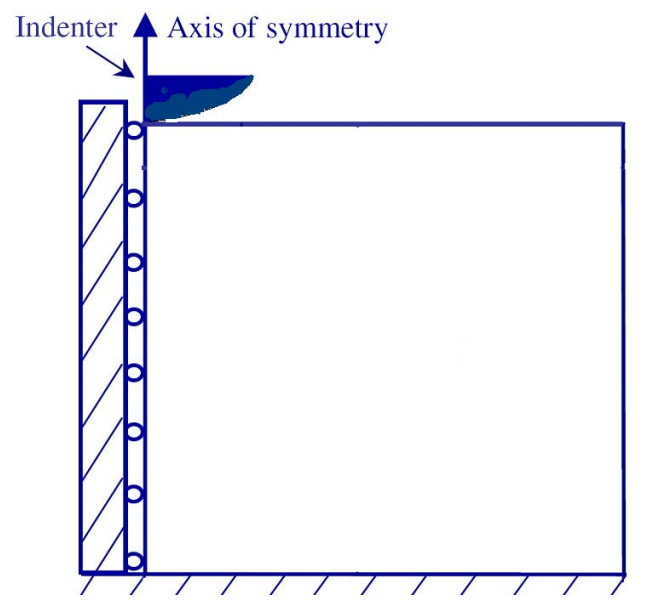

Fig. 3. Boundary conditions for FE model in nanoindentation model.

\section{RESUlTS AND DisCUSSION}

The result with frictionless contact and contact with friction coefficient 0.1 and 0.3 is shown in Fig. 4 imply that the assumption of frictionless contact is acceptable. The load displacement curve with different radius for indenter is shown in Fig. 5 As shown the load, required for $5000 \mathrm{~nm}$ depth indentation, increase with decreasing the indenter radius.

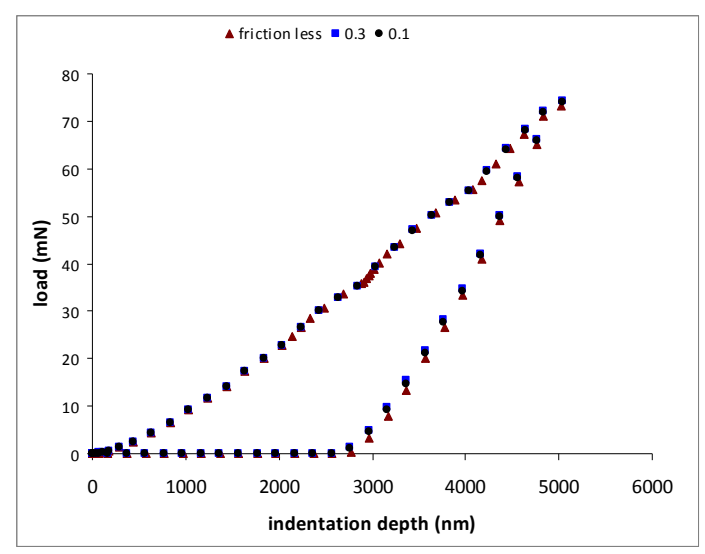

Fig. 4. Effect of friction coefficient.

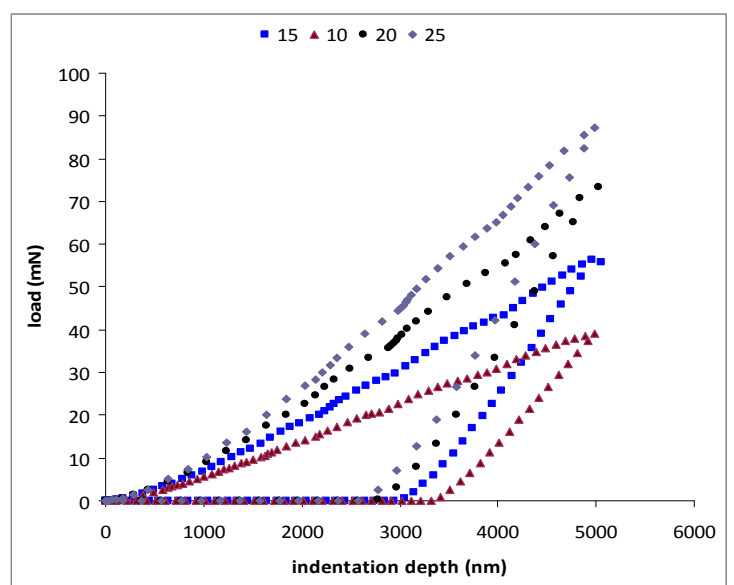

Fig. 5. Effect of indenter radius.

Fig. 6 shows the equivalent stress in the specimen when the indenter penetrate it in depth $5000 \mathrm{~nm}$ and fig. 7 show the stress couture in the unloading stage. One parameter that may be effective on results is specimen size. Here the load displacement curve for different specimen size $(8,10,12$, $14 \& 16$ times greater than penetration depth) is shown in Fig. 8. As shown the diagram has no significant changes when the ratio of specimen width to indentation depth is more than 10 , So 10 is selected. It is worth mentioning that the mesh size near the indentation region has great effect in results, so it should be as fine as possible. The results show that not considering friction is a reasonable assumption.

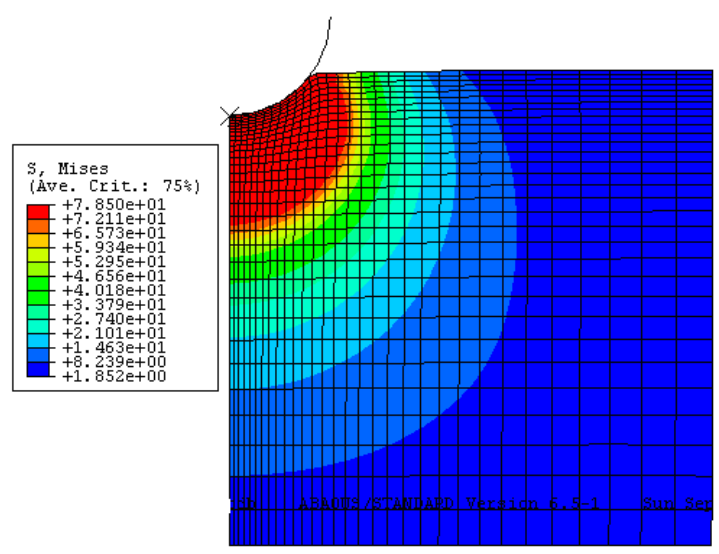

Fig. 6. Stress couture in depth $5000 \mathrm{~nm}$.

It should be noted that FEM model does not only depend on the accuracy and robustness of the technique used, but also on the accuracy of the elastic constants. However, these constants are not known very precisely. Even in reasonably well exfoliated clay nanocomposites the platelets can be made up of a number of intercalated silicate sheets and the spacing of the silicate sheets affects the Young's modulus and weight/volume relationship of the effective particle. The distribution of the aspect ratio of the clay particles is usually wide and so the appropriate aspect ratio for any model is uncertain. Moreover, the distribution of clay particles is far from uniform. The clay particles are usually not fully dispersed so that in typical epoxy/clay nanocomposites there are clusters of high particle concentration dispersed in a matrix of low particle concentration. It has been shown that stiffness of clustered composite is less than that of the same volume fraction of particles that are uniformly dispersed [12]. 
Thus, the accuracy of the modeling of tensile behavior of the nanocomposite is crucially dependent on the properties of the effective particle.

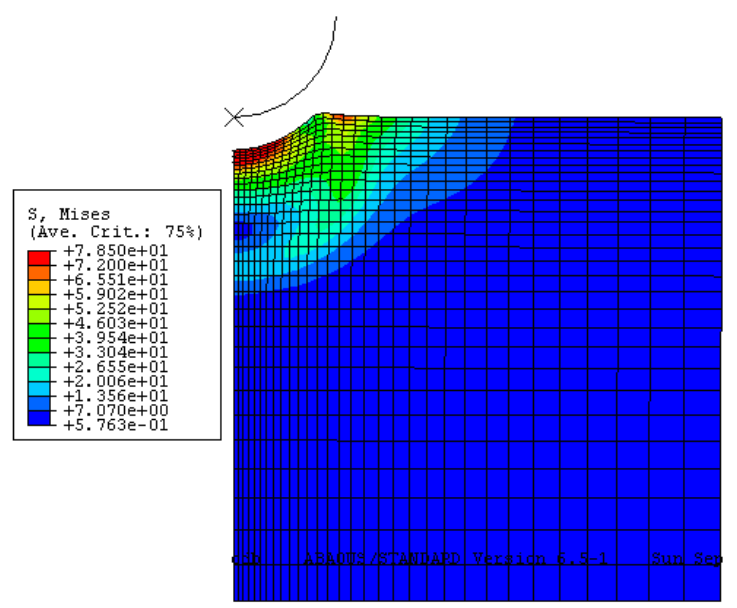

Fig. 7. Stress couture in unloading.

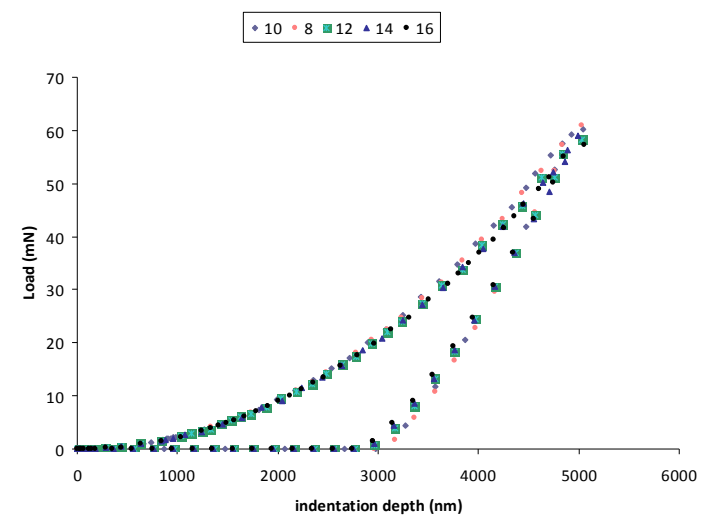

Fig. 8. Effect of specimen width.

\section{CONCLUSIONS}

In this work, representative volume element method and finite element method was successfully utilized to simulate spherical nanoindentation process of nanocomposites. The results show that the assumption of frictionless contact is acceptable. Also load, required indentation, increases with decreasing the indenter radius. Comparison of these simulated results with experimental results demonstrated that the continuum based FE approach has the capability to determine the load-depth response of a submicrometer indentation test.

\section{REFERENCES}

[1] A. Usuki, Y. Kojima, M. Kawasumi, A. Okada, Y. Fukushima, T. Kurauchi, et al."Synthesis of nylon 6-clay hybrid," Journal of Materials Research, vol. 8, pp.1179.D, 1993.

[2] Y. Kojima, A. Usuki, M. Kawasumi, A. Okada, Y. Fukushima, T. Kurauchi, et al."Mechanical properties of nylon 6-clay hybrid," Journal of Materials Research, vol. 8, pp. 1185, 1993.

[3] Y. Kojima, A. Usuki, M. Kawasumi, A. Okada, T. Kurauchi, O. Kamigaito, "Sorption of water in nylon 6-clay hybrid," Journal of Applied Polymer, vol. 49, pp.1259, Science 1993..

[4] S. Ray, Fertig III, and R. M. Garnich, "Influence of Constituent Properties and Microstructural Parameters on the Tensile Modulus of a Polymer/Clay Nanocomposite," Composites science and technology, vol. 64 , pp.2577-2588, 2004

[5] J. D.Bressan, A. Tramontin, and C. Rosa, "Modeling of nanoindentation of bulk and thin film by finite element method," Wear vol. 258, pp. 115-12, 2004.

[6] W. Chen, et al., "Influence of indenter tip roundness on hardness behavior in nanoindentation," Materials Science and Engineering A pp. 445-446, pp. 323-327, 2007.

[7] K. D. Bouzakisa, N. Michailidisa, and G. Erkensb, "Thin hard coatings stress-strain curves determination through a FEM supported evaluation of nanohardness results," Surface and Coatings Technology, pp. $142144,102109,2001$.

[8] Y. Hu, et al., "Nanoindentation studies on Nylon 11/clay nanocomposites," Polymer Testing, vol. 25, pp. 492-497, 2006.

[9] S. Lu, W. C. Tjiu, and T. Liu, "Nanoindentation and morphological studies on injection-molded nylon-6 nanocomposites," Polymer, vol. 46, pp. 11969-11977, 2005.

[10] L. Shen, I. Y. Phang, L. Chen, T. X. Liu, and K. Y. Zeng, " Nanoindentation and morphological studies on nylon 66/organoclay nanocomposites. II. Effect of strain rate," Polymer, pp. 8221-8229, 2004.

[11] M. Saadatfar and A. Soleimani, "Effective Parameters on the Elastic Modulus of Polymer/Clay Nanocomposite," submitted to ICMENS 2011

[12] J. J. Luo and M. Daniel, "Processing and Characterization of Epoxy/Clay Nanocomposites," Composites Science and Technology, vol. 63, pp.1607, 2003.

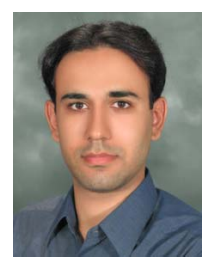

Mahdi Saadatfar was born in Qom /Iran on 1983. He received the diploma Degree in physics and mathematics from high School, Qom/Iran in 2001 and the BSc. degrees in Mechanical Eng. Kashan University, Kashan, Iran and Ms. Mechanical eng, University of Tehran, Tehran in 2008. He is currently a Ph.D. student at the School of Mechanical Engineering at K. N. Toosi University of Technology, Iran. Mr. Saadatfar's research interests are in the area of piezoelectric Materials, Polymer/Clay nanocomposites and Finite element modeling. He has several published paper about piezoelectric materials and Finite element modeling of nanocomposites.

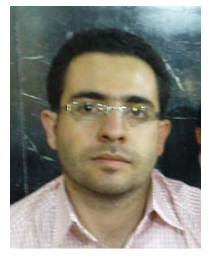

Ali Soleimani, was born in Isfahan /Iran on 1985 . $\mathrm{He}$ received the diploma Degree in physics and mathematics from high School, Isfahan/Iran in 2002 and the BSc. degrees in Mechanical Eng. Yazd University, Yazd, Iran and Ms. Mechanical eng, University of Tehran, Tehran in 2008. He is currently a Faculty member in Faculty of Mechanical Engineering at Islamic azad University, Najafabad Branch, Isfahan, Iran. Mr. Soleimani's research interests are in the area of vibration and condition monitoring. 